\title{
Comparative recognition by human IgG antibodies of recombinant proteins representing three asexual erythrocytic stage vaccine candidates of Plasmodium vivax
}

\author{
Mayara B Barbedo, Ricardo Ricci, Maria Carolina S Jimenez, Maristela G Cunha*, \\ Syed S Yazdani**, Chetan E Chitnis ${ }^{* *}$, Mauricio M Rodrigues ${ }^{* * *}$, Irene S Soares ${ }^{+}+$
}

\author{
Departamento de Análises Clínicas e Toxicológicas, Faculdade de Ciências Farmacêuticas, Universidade de São Paulo, \\ Av. Prof. Lineu Prestes, 580, Cidade Universitária, 05508-900 São Paulo, SP, Brasil *Departamento de Patologia, \\ Centro de Ciências Biológicas, Universidade Federal do Pará, Belém, PA, Brasil ** Malaria Research Group, International Centre for \\ Genetic Engineering and Biotechnology, New Delhi, India *** Departamento de Microbiologia, Imunologia e Parasitologia, \\ Escola Paulista de Medicina, Universidade Federal de São Paulo, São Paulo, SP, Brasil
}

In previous immuno-epidemiological studies of the naturally acquired antibody responses to merozoite surface protein-1 (MSP-1) of Plasmodium vivax, we had evidence that the responses to distinct erythrocytic stage antigens could be differentially regulated. The present study was designed to compare the antibody response to three asexual erythrocytic stage antigens vaccine candidates of $\mathrm{P}$. vivax. Recombinant proteins representing the $19 \mathrm{kDa} C$-terminal region of MSP-1(PvMSP $\left.{ }_{19}\right)$, apical membrane antigen $n-1$ ectodomain (PvAMA-1), and the region II of duffy binding protein (PvDBP-RII) were compared in their ability to bind to IgG antibodies of serum samples collected from 220 individuals from the state of Pará, in the North of Brazil. During patent infection with P. vivax, the frequency of individuals with IgG antibodies to PvMSP ${ }_{19}, P v A M A-1$, and PvDBP-RII were 95, 72.7, and $44.5 \%$ respectively. Although the frequency of responders to PvDBP-RII was lower, this frequency increased in individuals following multiple malarial infections. Individually, the specific antibody levels did not decline significantly nine months after treatment, except to $P v M S P 1_{19}$. Our results further confirm a complex regulation of the immune response to distinct blood stage antigens. The reason for that is presently unknown but it may contribute to the high risk of re-infection in individuals living in the endemic areas.

Key words: malaria - Plasmodium vivax - merozoite antigens - IgG antibody response

Plasmodium vivax is the second most prevalent specie that causes malaria in humans (Mendis et al. 2001). In Brazil, P. vivax was responsible by approximately $75 \%$ of 597,907 cases reported in 2005 (Secretaria de Vigilância em Saúde, Ministério da Saúde). In spite of its high prevalence in Brazil as in many other parts of the world, the immunological mechanisms operating in individuals exposed to $P$. vivax have been very poorly explored.

In recent years, we have carried out several immunological studies using malaria recombinant proteins corresponding to the $P$. vivax merozoite surface protein 1 (MSP-1) and apical membrane antigen 1 (AMA-1) (Soares et al. 1997, 1999, Rodrigues et al. 2003, 2005). These immuno-epidemiological studies on naturally ac-

Financial support: Fapesp (04/00768-7), The Millennium Institute for Vaccine Development and Technology (CNPq - 420067/20051). MBB, RR, MMR, and ISS are supported by fellowships from CNPq. MCSJ is supported by fellowships from Fapesp.

+Corresponding author: isoares@usp.br

Received 12 February 2007

Accepted 24 April 2007 quired immunity to merozoite proteins are of particular importance as these MSP-1 and AMA-1 are being intensively studied as a candidate for development of a vaccine against malaria (Good 2005). So far, we found that a high frequency of individuals from the Brazilian Amazon area, where $P$. vivax is endemic, had $\operatorname{IgG}$ antibodies to recombinant proteins based on $19 \mathrm{kDa}$ C-terminal region of MSP-1 (PvMSP1 19 ) (Soares et al. 1997, 1999, Rodrigues et al. 2003) and the AMA-1 ectodomain (PvAMA-1) (Rodrigues et al. 2005, Oliveira et al. 2006).

As a continuation of our immuno-epidemiological studies in Brazil, in the present study, we compared the IgG antibody response to recombinant proteins based on three asexual erythrocytic stage antigens vaccine candidates of $P$. vivax, including the region II of the duffy binding protein (PvDBP-RII).

\section{MATERIALS AND METHODS}

Human serum samples - Serum samples were collected during the period from 1995 to 1999 from 220 patients living in endemic areas for malaria in the state of Pará, in the North of Brazil. At the time of blood collection, these subjects were positive for $P$. vivax by conventional thick blood smears microscopic examination. Details on the study area, patient age and diagnosis have been reported previously (Soares et al. 1997, 1999, Rodrigues et al. 2003, 2005, Oliveira et al. 2006). Only individuals whom precise information on the number of $P$. vivax malaria episodes was available were used to es- 
tablish a correlation between the number of malarias and the frequency of responders to recombinant proteins. A second group of individuals $(\mathrm{n}=33)$ donated blood nine months after being treated for malaria. Those individuals did not report subsequent malaria infections in that period. A third group of individuals was healthy adult volunteers selected among blood donors from the city of São Paulo, state of São Paulo, in the Southeastern of Brazil $(n=26)$. Malaria is not present in this part of the country and these individuals had never had malaria or traveled to malaria endemic areas. The Ethics Committee of the University of São Paulo has approved our studies.

Recombinant proteins

$P v M S P 1_{19}$ - The recombinant protein PvMSP1 19 represents the $19 \mathrm{kDa} C$-terminal region of the $P$. vivax MSP-1. This protein was expressed in Escherichia coli and purified as publish elsewhere (Cunha et al. 2001).

PVAMA-1 - The recombinant representing the PvAMA-1 ectodomain was made as already reported (Rodrigues et al. 2005).

$P v D B P-R I I$ - The recombinant protein PvDBP-RII represents region II of $P$. vivax DBP. This protein was expressed in $E$. coli and purified as previously described (Singh et al. 2001).

ELISA for detection of human IgG antibodies - Human IgG antibodies against PvMSP1 19 , PvAMA-1, and PvDBP-RII were detected by ELISA as described (Rodrigues et al. 2003, 2005). The results were expressed as index of reactivity (IR $=\mathrm{OD}_{492}$ values of test sample divided by the value of the cutoff). Cutoff points were set at three standard deviations above the mean $\mathrm{OD}_{492}$ of sera from 26 individuals, unexposed to malaria, from the city of São Paulo. Values of IR $\geq 1.0$ were considered as positive.

Statistical analysis - Differences between proportions of responders were analyzed using the Chi-square test. Comparison of antibody level (IR) of independent samples was performed using one-way analysis of variance (ANOVA). Wilcoxon Signed Rank test was used to compare dependent samples.

\section{RESULTS}

Comparison of $\operatorname{IgG}$ antibody response of individuals during patent $P$. vivax infection to recombinant proteins representing $M S P 1_{19}, A M A-1$, and DBP-RII of $P$. vivax - Initially, we compared the IgG antibody response of individuals during acute infection to the recombinant proteins representing PvMSP1 ${ }_{19}$, PvAMA-1, and PvDBP-RII. The frequency of individuals with $\mathrm{IgG}$ antibodies to all three proteins during patent infection was only $37.7 \%$. No response to any antigen was observed in only $2.7 \%$ of individuals. Fig. 1 shows the frequency of responders against each recombinant protein. The results showed that the frequency of individuals that presented $\mathrm{IgG}$ antibodies to recombinant proteins PvMSP1 19 , PvAMA-1, and PvDBP-RII were 95, 72.7, and $44.5 \%$, respectively. These data confirmed that all three proteins are immunogenic during natural infections. The prevalence of antibodies against PvMSP1 19 was significantly higher than the prevalence against PvAMA-1 (Chi-Square test, $P<0.0001$ ). The prevalence for both were higher than those observed for PvDBPRII (Chi-Square test, $P<0.0001$ ).

When we compared the IR values from individual serum samples, we observed that values obtained for PvMSP $_{19}$ were higher than all other groups (Table I). The IR values to PvDBP-RII were lower than those observed to PvAMA-1 (One Way ANOVA, $P<0.0001$ ).

We then determined whether there was a correlation between the frequency of $\mathrm{IgG}$ antibodies and episodes of $P$. vivax infection. For this purpose, the sera of the 213 individuals were separated in two groups: (i) pri-

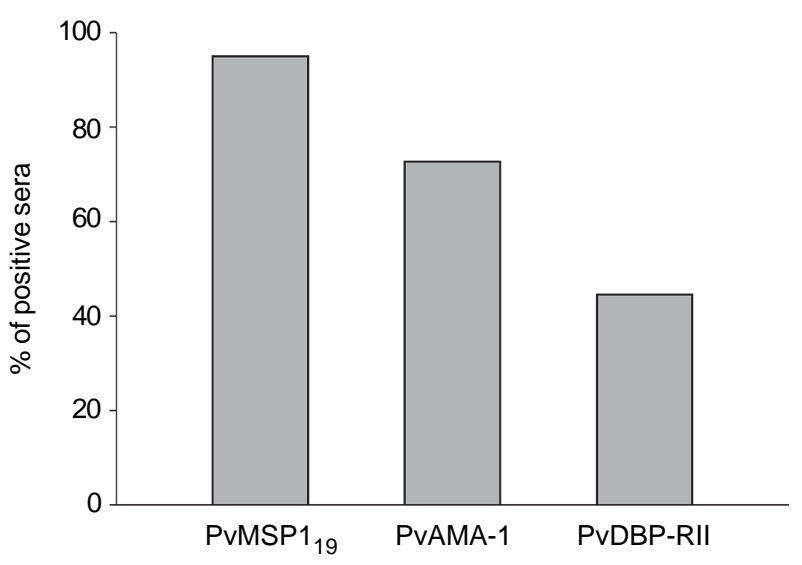

Fig. 1: antibody response to recombinant proteins corresponding to the MSP $1_{19}$, AMA-1, and DBP-RII of Plasmodium vivax during patent $P$. vivax infection. Serum samples were tested at 1:100 serum dilution. Percentage of responders was estimated from 220 individuals and corresponds to those serum samples that presented $\mathrm{OD}_{492}$ values 3 standard deviations above the average $\mathrm{OD}_{492}$ obtained from serum samples of 26 healthy individuals never exposed to malaria. Values of IR $\geq 1.0$ were considered as positive.

TABLE I

Magnitude of the IgG antibody response in 220 individuals with patent Plasmodium vivax malaria

\begin{tabular}{lcc}
\hline $\begin{array}{l}\text { Recombinant } \\
\text { protein }\end{array}$ & IR & IgG antibody level \\
\hline PvMSP1 $_{19}$ & 11.41 & $10.73-12.09$ \\
PvAMA-1 $_{\text {PvDBP-RII }}$ & 3.44 & $2.96-3.92$ \\
& 1.46 & $1.23-1.69$ \\
\hline
\end{tabular}

The antibody level was expressed as mean of index reactivity (IR, $95 \% \mathrm{CI}$ ) that corresponds to $\mathrm{OD}_{492}$ value of test sample obtained at 1:100 serum dilution divided by the value of the cutoff. The cutoff value was the mean $\mathrm{OD}_{492}$ value plus three standard deviations of the 26 healthy individuals. Values of IR $\geq 1.0$ were considered as positive. 
mary-infected, individuals with no previous malaria episodes $(n=145)$ and (ii) individuals with one or more previous malaria episodes $(n=68)$. We found that the frequency of responders to PvMSP $1_{19}$ did not change significantly when we divided them in primary and multiple-infected (Chi-Square test, $P>0.05$ ) confirming data previously obtained by our group that showed that antibody response against PvMSP1 19 was established after a single exposure to malaria (Rodrigues et al. 2003, 2005). In contrast, the frequency of serum samples containing IgG against PvAMA-1 and PvDBP-RII was significantly lower in group primary-infected when compared with the group multi-infected individuals (ChiSquare test, $P<0.0001$ ), suggesting that the seroconversion to these proteins occurs after multiple exposures to malaria infection (Fig. 2). However, no difference was observed between the frequency of positive sera during the second/third or more malaria episodes, showing that specific IgG responses to both antigens developed after few malaria episodes (data not shown).

Longevity of the $\operatorname{Ig} G$ antibody response to recombinant proteins representing $M S P 1_{19}, A M A-1$ and $D B P$ RII of $P$. vivax - In earlier studies, we observed that the IgG antibody titers to recombinant proteins representing the $\mathrm{N}$ - and $\mathrm{C}$-terminal regions of $P$. vivax MSP-1 declined significantly after treatment (Soares et al. 1999). Using serum samples collected during patent infection and nine months after treatment (paired groups, $\mathrm{n}=33$ ), we compared the frequency of responders and the anti-

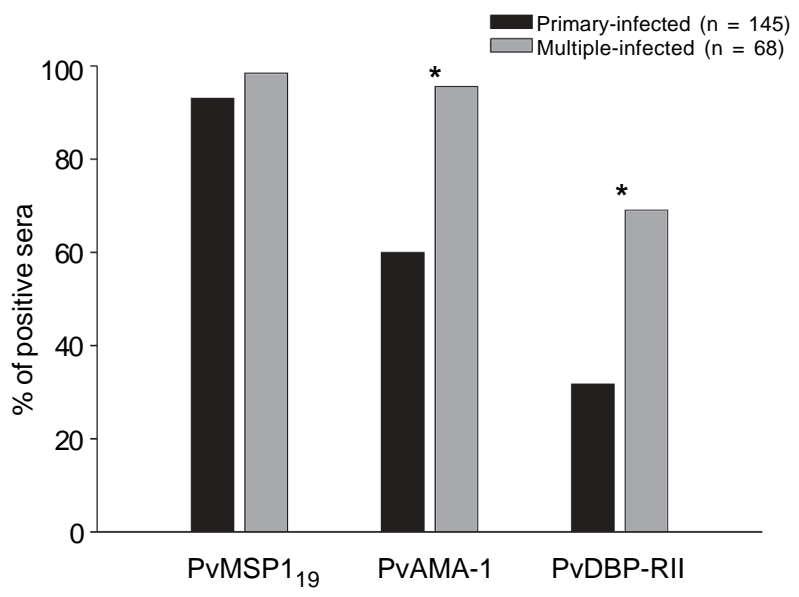

Fig. 2: association between the percentage of responders that recognize each recombinant protein and the number of previous malaria episodes. A total of 213 individuals were grouped according to the number of episodes of Plasmodium vivax malaria. The number of individuals of each group was 145 and 68 from primary and multiple infected people, respectively. Percentage of responders was estimated according to Fig. 1. Asterisks indicate frequency of responders statistically significant among individuals primary-infected and with multiple infections. body levels (estimated by $\mathrm{OD}_{492}$ ) to the different recombinant proteins. The proportions of individuals with antibodies specific to PvAMA-1 and PvDBP-RII were not significant during the infection or after treatment (ChiSquare test, $P<0.05$ ). The low frequency of responders to PvDBP-RII can be explained by the fact that only primary infected individuals constitute this group. In contrast, the proportions of individuals with antibodies specific to PvMSP1 $1_{19}$ were statistically higher during infection than nine months after treatment (Chi-Square test, $P<0.0001$ ).

When the antibody levels of individuals who recognized each recombinant protein were estimated (Table II), we found that these levels were not significant during the infection and after treatment, except to PvMSP1 19 (Wilcoxon Signed Ranks test, $P<0.0001$ ).

\section{TABLE II}

Comparison of frequency and antibody serum sample levels of each recombinant protein among individuals with acute Plasmodium vivax infection and after treatment

\begin{tabular}{lcccc}
\hline \multicolumn{3}{c}{ Paired } & \multicolumn{3}{c}{ group $(\mathrm{n}=33)$} \\
\hline Recombinant & \multicolumn{3}{c}{ Patent infection } & \multicolumn{2}{c}{ After treatment } \\
protein & $\%$ & IR & $\%$ & IR \\
\hline PvMSP1 $_{19}$ & 100.0 & 7.81 & $57.6 *$ & $1.43 *$ \\
PvAMA-1 $^{*}$ & 81.8 & 2.04 & 87.9 & 1.73 \\
PvDBP-RII & 15.1 & 0.34 & 6.1 & 0.25 \\
\hline
\end{tabular}

Percentage of responders was estimated according to Fig. 1. The antibody level to each recombinant protein is expressed as the median of index reactivity (IR). Asterisks indicate frequency of responders or antibody levels statistically significant among individuals with acute infection and after treatment.

\section{DISCUSSION}

In the present study we compared the recognition by human IgG antibodies of recombinant proteins representing three asexual erythrocytic stage vaccine candidates of $P$. vivax (MSP1 19 , AMA-1, and DBP-RII). Initially, we confirmed that the frequency of individuals who presented IgG antibodies to PvMSP1 ${ }_{19}$ and PvAMA-1 during patent infection was high and comparable to the frequency observed in our recent studies (Rodrigues et al. $2003,2005)$. On the other hand, the frequency of responders to DBP-RII was significantly lower. One possible explanation for this fact could be the requirement of repeated exposures to parasite antigens in order to promote seroconvertion. Eventually, repeated $P$. vivax infections could significantly increase the frequency of responders, indicating that most individuals may become responders depending on the degree of exposure. To answer that question, we divided the individuals that informed the number of previous malaria episodes in two groups: primary-infected and individuals with one or 
more previous malaria episodes. Our analysis showed that the frequency of responders to PvAMA-1 and PvDBP-RII was higher in the multiple-infected. This data suggests that multiple infections provide a boost to the production of specific antibodies confirming recent observations obtained in other studies in distinct endemic areas of Brazilian Amazon (Ceravolo et al. 2005, Tran et al. 2005). In the case of PvMSP1 19 , specific IgG responses developed faster after a single exposition to malaria infection, also confirming previous observations of distinct groups that $\mathrm{PvMSP}_{19}$ is highly immunogenic during natural human infections (Park et al. 2001, Lim et al. 2002, Rodrigues et al. 2003, Morais et al. 2005, Wickramarachchi et al. 2007).

We also evaluated the persistence of antibody response to the different recombinant proteins in individuals exposed to $P$. vivax. We compared the presence of specific antibodies and their levels in a period of nine months following $P$. vivax infection in the absence of reinfection. During acute infection, eleven individuals (33.3\%) displayed antibodies to all three proteins. After treatment, all those individuals persisted serologically positive to at least one antigen. When evaluated each individual recombinant protein, in the majority of individuals, specific IgG antibodies did not diminish significantly in that period, except to PvMSP1 19 . This observation confirms our earlier description that during acute infection, there was a significant higher frequency of responders who had higher IgG antibody titers to PvMSP1 ${ }_{19}$ when compared to individuals few months after treatment for malaria (Soares et al. 1997, 1999). The significant decay of the number of positive sera and antibody levels to PvMSP $1_{19}$ contrasted with the fact that the frequency of responders to PvAMA-1, increased slightly after treatment. The vast majority of the individuals $(85.7 \%)$ that became serologically negative to PvMSP1 ${ }_{19}$ maintained detectable antibodies to PvAMA-1. Similar results were recently obtained in a distinct endemic area of Brazilian Amazon which showed that anti-PvAMA-1 antibodies persisted in $>40 \%$ of those subjects exposed to focal malaria eight months before (Morais et al. 2006).

The reason why the antibody production is differentially sustained to each recombinant antigen after treatment is currently unknown and deserves to be investigated further in the future. Effective treatment of malaria infections has been shown to lead to rapid decline in antibody concentrations. In spite of that, memory B cells can persist in the absence of antigen and can be rapidly reactivated on reinfection (reviewed by Struik \& Riley, 2004).

In summary, our results show that all three vaccine candidates are immunogenic molecules during natural malaria infections and these recombinant proteins may be useful tools to perform immuno-epidemiological studies in malaria endemic areas and to further studies towards the vaccine potential of these proteins.

\section{ACKNOWLEDGEMENTS}

To Vanessa Carvalho Tavares for technical assistance.

\section{REFERENCES}

Ceravolo IP, Bruna-Romero O, Braga EM, Fontes CJ, Brito CF, Souza JM, Krettli AU, Adams JH, Carvalho LH 2005. AntiPlasmodium vivax duffy binding protein antibodies measure exposure to malaria in the Brazilian Amazon. Am J Trop Med Hyg 72: 675-681.

Cunha MG, Rodrigues MM, Soares IS 2001. Comparison of the immunogenic properties of recombinant proteins representing the Plasmodium vivax vaccine candidate MSP $1_{19}$ expressed in distinct bacterial vectors. Vaccine 20:385-396.

Good MF 2005. Vaccine-induced immunity to malaria parasites and the need for novel strategies. Trends Parasitol 21: 29-34.

Lim KJ, Park JW, Sohn MJ, Lee S, Oh JH, Kim HC, Bahk YY, Kim YS 2002. A direct sandwich ELISA to detect antibodies against the $\mathrm{C}$-terminal region of merozoite surface protein 1 could be a useful diagnostic method to identify Plasmodium vivax exposed persons. Parasitol Res 88: 855-860.

Mendis K, Sina BJ, Marchesini P, Carter R 2001. The neglected burden of Plasmodium vivax malaria. Am J Trop Med Hyg 64: 97-106.

Morais CG, Soares IS, Carvalho LH, Fontes CJF, Krettli AU, Braga EM 2005. IgG isotype to C-terminal $19 \mathrm{kDa}$ of Plasmodium vivax merozoite surface protein 1 among subjects with different levels of exposure to malaria in Brazil. Parasitol Res 95: 420-426.

Morais CG, Soares IS, Carvalho LH, Fontes CJF, Krettli AU, Braga EM 2006. Antibodies to Plasmodium vivax Apical Membrane Antigen-1 are long-lasting and correlate to the malaria transmission intensity. Am J Trop Med Hyg 75: 582-587.

Oliveira TR, Fernandez-Becerra C, Jimenez MCS, Del Portillo HA, Soares IS 2006. Evaluation of the acquired immune responses to Plasmodium vivax VIR variant antigens in individuals living in malaria-endemic areas of Brazil. Malar J 5: 83.

Park JW, Moon SH, Yeom JS, Lim KJ, Sohn MJ, Jung WC, Cho YJ, Jeon KW, Ju W, Ki CS, Oh MD, Choe K 2001. Naturally acquired antibody responses to the C-terminal region of merozoite surface protein 1 of Plasmodium vivax in Korea. Clin Diagn Lab Immunol 8: 14-20.

Rodrigues MHC, Cunha MG, Machado RLD, Ferreira-Jr OC, Rodrigues MM, Soares IS 2003. Serological detection of Plasmodium vivax malaria using recombinant proteins corresponding to the $19-\mathrm{kDaC}$-terminal region of the merozoite surface protein-1. Malar J 2: 39.

Rodrigues MHC, Rodrigues KM, Oliveira TR, Comodo AN, Rodrigues MM, Kocken CH, Thomas AW, Soares IS 2005. Antibody response of naturally infected individuals to recombinant Plasmodium vivax apical membrane antigen-1. Int J Parasitol 35: 185-192.

Secretaria de Vigilância em Saúde-SVS, Ministério da Saúde. http://www.saude.gov.br

Singh S, Pandey K, Chattopadhayay R, Yazdani SS, Lynn A, Bharadwaj A, Ranjan A, Chitnis C 2001. Biochemical, biophysical, and functional characterization of bacterially expressed and refolded receptor binding domain of Plasmodium vivax Duffy-binding protein. J Biol Chem 276: 1711117116.

Soares IS, Cunha MG, Silva MN, Souza JM, Del Portillo HA, Rodrigues MM 1999. Longevity of naturally acquired anti- 
body responses to the $\mathrm{N}$ and C-terminal regions of Plasmodium vivax merozoite surface protein 1. Am J Trop Med Hyg 60: 357-363.

Soares IS, Levitus G, Souza JM, Del Portillo HA, Rodrigues MM 1997. Acquired immune responses to the $\mathrm{N}$ - and C-terminal regions of Plasmodium vivax merozoite surface protein 1 in individuals exposed to malaria. Infect Immun 65: 1606-1614.

Struik SS, Riley EM 2004. Does malaria suffer from lack of memory? Immunol Rev 201: 268-290.

Tran TM, Oliveira-Ferreira J, Moreno A, Santos F, Yazdani SS,
Chitnis CE, Altaman JD, Meyer EV, Barnwell JW, Galinski MR 2005. Comparison of IgG reactivities to Plasmodium vivax merozoite invasion antigens in a Brazilian Amazon population. Am J Trop Med Hyg 73: 244-255.

Wickramarachchi T, Illeperuma RJ, Perera L, Bandara S, Holm I, Longacre S, Handunnetti SM, Udagama-Randeniya PV 2007. Comparison of naturally acquired antibody responses against the C-terminal processing products of Plasmodium vivax Merozoite Surface Protein-1 under low transmission and unstable malaria conditions in Sri Lanka. Int J Parasitol 37: 199-208. 
